\title{
DIE ANALYSE DER JUGENDSPRACHE IM DEUTSCHEN
} KINOFILM “DOKTORSPIELE” AUS DEM JAHR 2014

\author{
Duma Elfrida Manurung \\ Ahmad Bengar Harahap \\ Risnovita Sari
}

\begin{abstract}
AUSZUG
Das Ziel dieser Untersuchung ist es, um die Jugendsprachen in dem Film "Doktorspiele" von Marco Petry herauszufinden und die Jugendsprache stellt in dem Film "Doktorspiele" dar. Die Datenquelle dieser Untersuchung ist die Dialoge im Film "Doktorspiele" von Marco Petry. Die Ergebnisse dieser Untersuchung stellt 33 jugendsprachliche Audrücke in dem Film "Doktorspiele"dar, die durch Theorie von Helmut Henne in Gross (2000:5) analysiert wird. Das Nutzen dieser Untersuchung ist es, um Informationen über die Bedeutung der Jugendsprache zu liefern und es ist neue, interresante Methode mit einem Film Deutsch zu lernen. Die Methode dieser Untersuchung wird deskriptive qualitative Methode verwendet. Diese Untersuchung wird in der Digital Library an der staatlichen Universität von Medan durchgeführt.
\end{abstract}

\section{Schlüsselwörter : Jugendsprache und Film}

\section{EINLEITUNG}

Die Sprache ist ein Kommunikationsmittel, das verwendet wird, um Menschen miteinander zu verbinden. Sprache ist ein Teil der Identität, die variieren kann. Sprache kann Informationen, Meinungen, und Expressionen ausdrücken. Mitder Sprache kann man den Blickwinkel zeigen, den Ursprung die Nation und Land, Bildung und sogar die Beschaffenheit. Dieses Phänomen führt zu Sprachvariation. Kridalaksana im Purba (1996:37) erklärt, dass Sprachvariationen in diesen Themen eine Variation nach unterschiedliche Verwendungnach den Sprecher Beziehungen sind.Sprache ist universell und flexibel. Das bedeutet, dass es Entwicklung oder der Sprachverschiebung passieren kann. Dieses Phänomen verursacht viele Sprachenneben der Standardsprache, nämlich Dialekt, Argon, Jargon, Slang und Jugendsprache. In der heutigen Zeit findet die Verbereitung der Jugendsprache nicht mehr nur von Mund zu Mund statt, sondern auch kann durch Soziale Netzwerke, Lieder und Filme. Der Film ist das einfachste Medium, um dasInteresse der Öffentlichkeit zu gewinnen.Man kann auch einen Film als Lernmedium benutzen, der eine Nachricht oder ein bestimmtes Lehrthema vermittelt. Der Film unterhält den Betrachter nicht nur als Audio, sondern auch Visuell.Diese Untersuchung befasstsich 
mit Jugendsprache im Film eingeführt. Im täglichen Umgang wird Jugendsprache bewertet, um das Vokabular reicher $\mathrm{zu}$ machen. In einer offiziellen Situation verwendet Jugendlichen manchmal die Jugendsprache. Der Grund für Jugendliche, eine Jugendsprache einzuführen, ist Deutsch kommunikativer zu sprechen, aber das Interesse der Jugendliche ist etwas negative zu lernen, zum Beispiel: "Fuck!" ist Schimpfwort, "Blödi!" bedeutet Blödmann (Jugendsprache in dem Film Doktorspiele). Manchmal verwenden Jugendlichen die Jugendsprache, um ihre Freunde einfach nachzuahmen und versteht nicht die Bedeutung. Dieses Phänomen ist der HintergrundJugendsprache zu untersuchen. Die Jugendliche kann mit der Atmosphäre, Ort und der Gesprächspartner anpassen, um die Jugendsprache zu benutzen, wenn sie die Bedeutung der Jugendsprache kennen, um Missverständnisse zu vermeiden.

\section{THEORETISCHE GRUNDLAGE}

\section{Der Begriff der Jugendsprache}

Nach Ansicht von Helmut Henne (im Glas2004: 2) ist Jugendsprache eine Sprechund Schreibweise, die zur jugendlichen Sprachprofilierung und zur Identitätsfindung dient. Jugendsprache ist ein Stil, der durch die Entwicklung oder Änderung einer Vielzahl von Sprachen verursacht wird.Jugendsprache hat keine eindeutige stilistische Struktur. Die meisten Wörter in der Jugendsprache kommen aus Übersetzungen, Abkürzungen und Wortspielen . Es gibt aber auch Wörter, deren Ursprung schwer zu finden ist. Es gibt Jugendsprache, weil die Standardsprache immer komplizierter und fachspezifischer wird und selbst von vielen Erwachsenen heute weder beherrscht, noch wirklich ernst genommen wird. In seinem Buch „Jugend und ihre Sprache” schreibt er, dass Jugendsprache eineStandardsprache schöpferisch abwandelt, ,stereotypisiert" und ,spezifische Formen ihres sprachlichen Spiels" pflegt (Eichler 2008:5). Das Vokabular im Slang ist unbeständig, weil Slang nicht zeitlich begrenzt und wandelbar ist. Ursprünglich war Slang eine geheime Sprache zwischen den Mitgliedern einer Gruppe von Dieben, Taschendieben, oder Banditen, die sich weiterentwickelt hat und mit neuen Elementen von Jugendlichenuntereinander verwendet wird (Poedjosoedarmo, 2003: 66) im 
(http://etd.repository.ugm.ac.id). Im Erlernen einer Sprache ist Jugendsprache eine interessante Sache. Jugendsprache im täglichen Gespräch ist nützlich um kommunikativer und wirkt entspannter. Das bedeutet, dass Jugendsprache nur in informellenSituationen verwendet wird. Jugendlichensind wirklich wie „Aufständische“ und das wird in der Rede reflektiert. Der Aufstand wird durch die Verwendung von Non-Standard-Sprache und die Schaffung von Non-StandardFormen ausgedrückt (Sumarsono 2012: 159). Daher klingt das Vokabular und die Bedeutung von Jugendsprache häufig negativ und unhöflich. Zusätzlich kann Jugendsprache auchvon Abkürzungen und Akronyme gebildet werden, die neuer Begriff und fremd ist und es gibt nicht im Indonesischen Großen Wörterbuch (Mastuti, 2008:70) im (http://etd.repository.ugm.ac.id).

Es kann zusammengefasst werden, dass Jugendsprache die Sprache ist, die die Jugendlichen üblicherweise zur Sprachprofilierung und zur Identitätsfindung verwenden. Jugendsprache kommt aus dem Slang und wird in informellen Situationen verwendet. Die meisten Wörter in der Jugendsprache kommen aus Übersetzungen, Abkürzungen und Wortspielen.

\section{2 .Die Merkmaleder Jugendsprache}

Ein Merkmal der jugendlichen Sprache ist „Kreativität“. Diese Vielfalt bestehtnicht nur in der Sprache, sondern man kann sie auch Sozial sehen (sumarsono 2012:156).Unter der strukturellen Dimension des Jugendtons versteht Henne Sprüche, (Sprech-) Syntax, Redensarten, Wortbildungen, Wortschatz, Prosodik und Graphik mit folgenden jugendsprachlichen Merkmalen (in Gross 2000:5):

a) Eigenwillige Grüße, Anreden und Partnerbezeichnungen:

Tussi, Macker

b) Griffige Namen und Spruchwelten:

Schwachmat $=$ Dummkopf

Was läuft denn hier für ein Film? Ein Ausdruck der Erstaunens

c) Flotte Redensarten und stereotype Eloskeln:

Ganz cool bleiben/ alles easy/ etwas am Start haben

d) Metaphorische, zumeist hyperbolische Sprechweisen:

Sklaventreiber $=$ Lehrer, megageile Party $=$ gelungene Party

e) Repliken mit Entzückungs- und Verdammungswörtern:

Galaktisch = großartig Entzückungswort

Assig $=$ schlecht Verdammungswort

f) Lautwörterkommunikation:

Zuck zusammen Wurzelwörter für den Zustand des sich Erschreckens 
Basch $\longrightarrow$ Lautnachahmendes Wort für das Schlagen auf ein Schlagzeug

g) Prosodische, die Lautstruktur betreffende Sprechweisen:Wahnsinn

h) Wortbildungen (Neubedeutungen, Neuwörter), Worterweiterungen (Präfix-, Suffixbildung), Kurzwörter:

Keule Neubedeutung für Mädchen/Freundin

Mucke $\longrightarrow$ Neuwort für Musik

\section{UNTERSUCHUNGSMETHODE}

\section{A. Die Untersuchungsmethode}

In dieser Untersuchung wird die deskriptive qualitative Methode verwendet.

\section{B. Die Daten und die Datenquelle}

Die Daten in dieser Untersuchung sind die Dialoge im Film „Doktorspiele” und die Datenquelle ist der Film "Doktorspiele” von Marco Petry, der 97 Minuten lang ist.

\section{Der Untersuchungsort}

Diese Untersuchung wird in der Bibliothek an der Fakultat für Sprachen und Kunst an der staatlichen Universität von Medan durchgeführt.

\section{Die Technik der Datensammlung}

Für die Datensammlung wird die Theorien von Miles und Huberman (in Hutagalung2015:15) angewendet:

1. Der Film „Doktorspiele” wird angeschaut.

2. Die Dialoge in diesem Film werden schriftlich erfasst.

3. Die verwendete Jugendsprache wird identifiziert und markiert.

4. Die jugendsprachlichen Ausdrücke werden in einer Tabelle geordnet.

\section{E. Die Technik der Datenanalyse}

Für die Datensammlung wird die Theorien von Miles und Huberman (in Hutagalung2015:15) angewendet:

1. Die Triangulation wird durchgeführt.

2. Die Jugendsprache wird mithilfe der Merkmale der Jugendsprache von Henne in Gross (2000:5) analysiert.

3. Das Ergebnis der Analyse wird von einem Experten geprüft.

4. Zum Schluss wird eine Zusammenfassung geschrieben.

\section{DAS ERGEBNIS DER UNTERSUCHUNG}




\section{A. Die Jugendsprache in dem Film "Doktorspiele"}

Während der Untersuchung wurden 33 jugendsprachliche Ausdrücke in dem Film "Doktorspiele" von Marco Petry gefunden. Die Analyse der Jugendsprache im Film "Doktorspiele" wird nach der Merkmale der Jugendsprache von Henne in Gross (2000:5) gemacht. Im Folgenden wird die Verwendung von Jugendsprache im Film "Doktorspiele" von Marco Petry analysiert:

\section{Eigenwillige Grüße, Anreden und Partnerbezeichnungen:}

\section{a. Alter}

(895) Bobby : Andi, Alter, merkst du noch was?

Inder Jugendsprache ist das Wort "Alter" eine Anrede für Ehemann, Freund, männliche Person und kann auch als Anrede für Vater. Das Wort wird unabhängig vom Alter der gesprochenen Person benutzt und ist respektlos (www.duden.de). Das Wort “Alter” kommt aus Wortspiel. Im Hennes Modelle Jugendsprache wird das Wort "Alter" als eigenwillige Grü $\beta$, Anrede und Partnerbezeichung kategorisiert.

b. Ey!

(120) Harry : :'Ey, ey, hau ab. Hau ab!

In diesem Kontext funktioniert der Ausdruck als Anrede. In diesem Kontext wird das Wort "ey!" als Anrede in Hennes Modell der Jugendsprache kategorisiert. Das Wort "ey!" kommt aus Wortspiel, die Jugendlichen benutzen.

\section{c. Hi!}

(175) Jaromir : Andi! Alter Freund. Na? Hi, Mademoiselle. Kommt, rein.

Das Wort "Hi" ist freundschaftliche, familiäre. Das Wort "Hi" ist eine mündliche Begrüßung bei persönlichen Begegnungen und Telefongesprächen, bei denen der Angerufene weiß, wer ihn anruft (sodass auf eine Nennung des Namens verzichtet werden kann). Das Wort kommt etwa ab Mitte der 1990er (Jugendsprache ab Ende der 1960er) Jahre von Englisch (www.redensartenindex.de). Das Wort "hi" kommt aus Englisch. Das Wort "Hi” kategorisiert als Grü $\beta$ in Hennes Modelle der Jugendsprache.

\section{Griffige Namen und Spruchwelten:}

\section{a. Blödi}

(2) Lilli : Okay, du Blödi, dann halt erst ich. 
https://jurnal.unimed.ac.id/2012/index.php/studia/index

Das Wort "Blödi" bedeutet Dummkopf. Das Wort "Blödi" ist Wortspiel und wird in griffige Namen in Hennes Modell der Jugendsprache kategorisiert.

\section{b. Schwachmat}

(894) Bobby : Ich steh nicht auf Frauen. Unglaublich, dass Lilli sich in so 'nen Schwachmaten verliebt.

Das Wort "Schwachmat" bedeutet Beleidigung, ähnlich wie "Idiot" oder "Dummkopf". Angelehnt an "Schwachsinn" oder "Schwachkopf".Das Wort "Schwachmat" ist Wortspiel und wird in griffige Namen in Hennes Modell der Jugendsprache kategorisiert.

\section{Repliken mit Entzückungs- und Verdammungswörtern:}

a. Entzückungswort

\section{i. Cool}

(539) Katja :Cool. Kommst du heute Abend vorbei?

Cool ist ein Wort, das man eine steile Karriere bescheinigen kann. Neben "gut" hat cool in bestimmten Kontext noch immer die Bedeutung, (bewundernswert) lässing, beherrscht, souverän, dies entspricht dem englischen Original. Das Wort "cool” ist typische Beispiele der Jugendsprache der 80-er( www.anglizismen-sprachberatung.de). Das Wort "cool" kommt aus Übersetzung und wird als Entzückungswort in Henne Modell Jugendsprache kategorisiert.

\section{ii. Geil}

\section{(50) Harry : Geil?}

Das Wort "geil” kommt aus Wortspiel. Hier soll den Bedeutungswandel von "geil” erläutert werden.Im 12. Jahrhundert bedeutet das Wort kraftvoll, üppig. Heute bedeutet es "sexuell erregt" bzw. Das Wort "geil" ist Wortspiel und wird als Entzükungswort in Hennes Modell der Jugendsprache kategorisiert.

\section{iii. Krass}

(463) Harry

:Krass!

Die Bedeutung von "krass" schwankt nach Kontext und so kann dieser Begriff einerseits ein Zeichen von Betroffenheit sein "Boah, das ist krass!" anstelle von "Oh Gott!", "Wie erschütternd!”, “ Wie heftig!” oder auch Erstaunen sowie Bewunderung zum Ausdruck bringen wie in Dialog im Film "Doktorspiele" "Krass! Was ist passiert?" (www.sprachenudel.de). Die 
https://jurnal.unimed.ac.id/2012/index.php/studia/index

Bedeutung von "krass" zeigt, dass das Wort "krass" als Repliken mit

Entzükungswort in Hennes Modelle der Jugendsprache kategorisiert wird.

iv. Porno

(76) Harry : Der so porno ist, das du nie wieder einen anderen zum Wichsen brauchst, ne?

Das Wort "porno" in diesem Kontext bedeutet super. Dieses Wort "porno" ist Wortspiel und wird in Repliken mit Entzückungswort in Hennes Modell Jugendsprache kategorisiert.

\section{v. Spitze}

(793) Andi $\quad$ : Spitze.

Das Wort "Spitze" bedeutet großartig, hervorragend und deshalb Begeisterung, begeisterte Bewunderung (www.duden.de). Das Wort ist Jugendsprache, das im Entzückungswort kategorisiert wird.

\section{vi. Irre}

(610) Katja : Mein Bruder hat sich auch nicht getraut und der ist total irre.

Das Wort “irre" ist Jugendsprache seit den 50-er (www.50_jahre_jugendsprache.pdf). Die Jugendlichen benutzen das Wort"irre", um "sehr gut" zu sagen. Das Wort "irre" kommt aus Wortspiel, das in Repliken mit Entzückungswort kategorisiert wird.

vii. Super

(345) Lilli : Du singst doch super. Die Show kam an.

Das Wort "Super" kommtaus Wortspiel und wird als Repliken mit Entzückungswort in Hennes Modell der Jugendsprache kategorisiert.

\section{viii. Sü $\beta$}

(606) Katja : Nein,nein, das mein ich nicht, sondern dass du für mich gesungen hast.Das war echt süß. Ach so, das. 
Das Wort "Süß” trägt die Jugendliche, um Repliken mit Entzückungswort zu sprechen. In diesem Dialogue bedeutet das Wort “süß” nett, anziehnd, schön.

\section{ix. Voll}

(33) Harry : Der Labert die so voll, bist sie nicht mehr wissen,wo vorne und hinten ist.

Das Wort "voll” bedeutet besonders (Bekräftigung). Das Wort "voll” ist ein Wort, das die Jugendlichen benutzt, um "sehr gut" zu sagen (https://jannisandroutsopoulos.files.wordpress.com). Das Wort "voll" wird in Repliken mit Entzückungswort in Hennes Modelle der Jugendsprache kategorisiert.

\section{Lautwörterkommunikation}

\section{a. Boah}

(611) Katja : Also tschaui. Boah, bist du mutig.Andi?

Das Wort "Boah" ist jugendsprachliche Ausruf des Erstaunens, der Überaschung. Das Wort “Boah” ist Jugendsprache der 90-er Jahre. Das Wort "boah" kommt aus Wortspiel und wird in Lautwortkommmunikation in Hennes Modell der Jugendsprache kategorisiert.

b. Echt?

(109) Harry : echt? Wer denn? Diese Lilli?

Da fragt man vorsichtshalber noch mal nach, wenn man nicht sicher ist, ob etwas ernst gemeint war. Oder mit Ausrufezeichen zur Bestätigung (www.mundmische.de). Das Wort "echt" ist Wortspiel und wird als Lautwortkommmunikation in Henne Modell Jugendsprache kategorisiert.

\section{c. Wow}

(338) Lilli : Wow, bist ja echt eine große Hilfe.

Das Wort "Wow" wird in Lautwortkommunikation kategorisiert. Das Wort "Wow" hat keine deutliche Bedeutung. Es könnte ein Wort sein, um Überaschung zu zeigen. Das Wort "wow" wird als Lautwortkommmunikation in Henne Modell Jugendsprache kategorisiert.

\section{Wortbildungen(Neubedeutungen, Neuwörter), Worterweiterungen} (Präfix-, Suffixbildung), Kurzwörter:
a. Neubedeutungen
i. Bock haben
(592) Katja : Hast du Bock? 
Das Wort "Bock haben" bedeutet Lust haben. Das Wort ist Jugendsprache, entstanden etwa Ende der 1970er Jahre. Das Wort kommt von Wortspiel. Im Hennes Modelle Jugendsprache wird das Wort "Bock haben" als Neubedeutungkategorisiert und kommt aus Wortspiel. (www.redensartenindex.de).

\section{ii. Hammerparty}

(401) Andi

: Ansonsten war's doch 'ne Hammerparty

Das Wort "hammer" ist etwas richtig fetzt, rockt, reihaut und was richttig

Gutes eben (www.sprachnudel.de). Das Wort "Hammer" ist Jugendsprache, das von Wortspiel kommt. Die wahre Bedeutung von Hammer ist nicht etwas, sehr, besonders und richtig fetzt, sondern einWerkzeug, das häufig zum Nageln, ein Objekt zu fixieren, Metall zu schmieden und ein Objekt zu zerstören verwendet wird. In diesem Dialog Hammerparty bedeutet ein wunderbares Fest.

\section{iii. Latte}

(385) Harry : Alter, hast du 'ne Latte?

Das Wort "Latte" ist Jugendsprache, das in Neubedutung kategorisiert wird. Das Wort bekommt eine Neubedeutung von "Art von Trinken" zu "egal" oder das Wort bedeutet egal sein" und Das Wort "Latte" kommt aus Wortspiel.

\section{iv. Nudelsalat}

(15) Harry

:ist klar, Captain Sparrow. Über warmen Nudelsalat oder was?

Das Wort "Nudelsalat" bedeutet Party mit zu vielen männlichen Teilnehmern (100\% Jugensprache 2017, 2016:98). Dieses Wort "Nudelsalat" ist eigentlich Art von Essen. Aber in der Jugendsprache bekommt dieses Wort eine neue Bedeutung. Das Wort "Nudelsalat" kommt aus Wortspiel.

\section{v. Typ}

(866) Lilli : Nein.Warum? Ich weiß, wie gut du Bobby kennst, aber...Ich bin nicht sein Typ.

Das Wort "Typ" wird in Neubedeutung in Hennes Modell der Jugendsprache kategorisiert."Typ" kommt aus Wortspiel. Das Wort "Typ" beduetet wörtlich Art, etwas typische.Aber in diesem Kontext bezeichnet das Wort “Typ” einen jungen Mann. Das Wort “Typ” ist typische Beispiele der Jugensprache in den 50-er Jahren. 
https://jurnal.unimed.ac.id/2012/index.php/studia/index

\section{vi. Spinnen}

(386) Andi

\section{: Spinnst du?}

Das Wort "spinnen" ist Jugendsprache. Das Spinnen des Fadens hatte im Volksglauben etwas Geheimnisvolles, und man glaubte, die Spinnerin könne dem Faden Gutes und Böses, Freundliches und Geheimnisvolles einspinnen. Geläufig sind unter der Perspektive des Erzählens noch Begriffe wie etwas fort-/weiterspinnenund das Hirngespinst (eine verrückte, unrealistische Idee). Das Wort ist heutigen Jugensprache (www.redensarten-index.de).

b. Neuwörter

i. Cool

(539) Katja : Klar. Cool. Kommst du heute Abend vorbei?

Das Wort "Cool" kann auch als Neuwort in Hennes Modellder Jugendsparche kategorisiert wird. Dieses Wort stammt aus Englisch, das “gut”, "geil” bedeutet. Das Wort “cool” kommt aus Übersetzung. Das Wort "cool" ist ein typischesBeispiel der Jugendsprache der 80-er( www.anglizismen-sprachberatung.de).

\section{ii. Fake}

(97) Andi

: Das ist ein Fake.

Das Wort "Fake" ist Englisch für Schwindel. Das Wort "fake" kommt aus Übersetzung. Besonders im Internet weit verbreitet. "Das ist ein Fake!" meint Das kann nicht stimmen. Beispiel "Das ist Fake!" bezeichnet die Vermutung, dass an einer Information oder generell etwas nicht stimmt (www.sprachnudel.de)

\section{iii. Okay}

(278) Lilli : Bist du Okay?

Das Wort "Okay" bedeutet alles in Ordnung und stammt aus dem Englischen. Das Wort "Okay" wird in Wortbildungen (Neuwort) in Deutschen kategorisiert.

\section{iv. Party}

(172)Lilli : Bei ihr ist die Party heute.

Das Wort "Party" bedeutet Fest. Das Wort kommt aus dem Englischen, das bei Jugendlichen beliebt ist(http://lexikon der jugendsprache.pdf).

Das Wort wird im Wortbildung als Neuwort kategorisiert.

c. Suffixbildung 
https://jurnal.unimed.ac.id/2012/index.php/studia/index

\section{i. Ficken}

(33) Harry : "Wer ficken will, muss freundlich sein"

Das Wort "ficken"ist derbe Jugendsprache; Das Wort gilt als Bezeichnung für triebhaften und lieblosen Geschlechtsverkehr, dabei hängt der Grad der Tabuisierung stark vom kulturellen bzw. subkulturellen Umfeld ab (www.redensarten-index.de). Das Wort "ficken" kommt aus Englisch. "ficken" bekommt Endung -en von Wort "fick". Daswegen "ficken" wird als suffixbildung in Henne Modell Jugendsprache kategorisiert.

d. Kurzwörter

\section{i. Assi}

(102) Andi : Ich find's trotzdem assi.

Das Wort "Assi" ist eine verkürzte Form von "Assistent". Dieses Wort kommt aus Abkürzungen. "Assi" wird zum Beispiel oft in der universitären Umgebung benutzt, für den Assistenten des Professors, welcher meist für Vorlesungsfolien, Übungen bzw. Klausuren zuständig ist.Nicht zu verwechseln mit dem Vorsatz "asi" von "asozial", der "assi" ausgesprochen und daher auch oft so geschrieben wird. Das Wort "Assi" wird als Kurzwort in Hennes Modelle der Jugendsprache kategorisiert.

\section{ii. Auf jeden}

(21) Bobby

Der : artig. Auf jeden

Ausdruck "Auf jeden" bedeutet

unbedingt;sicher;durchaus;injedem Fall. Normalerweise sagt man, wenn man beim Sprechen abgeschweift war und nun wieder auf das eigentliche Thema kommt bzw. um die Kernaussage anzukündigen. (www.redensarten-index.de). Das Wort "auf jeden" ist ein Abkürzungswort von "auf jeden Fall”. Der Ausdruck "auf jeden" wird als Kürzwort in Henne Modell Jugendsprache kategorisiert.

\section{iii. Auf keinsten}

(59) Harry

\section{: Auf keinsten.}

Das Ausdruck "auf keinsten" ist die Kurzwort von "Auf keinen Fall", wo bei hier deutlich wird, dass Jugendliche sich auf keinen Fall von grammatischen Greanzen aufhalten lassen. Das Ausdruck "auf keinsten" kommt aus Abkürzung. Deswegen wird dieses Wort als Kurzwort in Hennes Modell der Jugendsprache kategorisiert.

iv. Mums

(48) Andi : Unsere Mums sind befreundet. 
https://jurnal.unimed.ac.id/2012/index.php/studia/index

Das Wort "Mums" ist Abkurzung für Mutter. Deswegen wird "auf keinsten"als Kürzwort in Henne Modell Jugendsprache kategorisiert. Die Jugendliche kürzt das Wort "Mutter" ab, es einfach auszudrucken.

\section{v. Spast}

(358) Andi : So ein Spast!

Das Wort ist Jugendsprache, derb, Schimpfwort; diskriminierend gegenüber Menschen, die an spastischen Behinderungen (verkrampfteSpannungszustände der Muskulatur - keine geistige, sondern eine körperliche Behinderung) leiden. Mindestens seit 1970 als Schimpfwort gebraucht (www.redensarten-index.de).

\section{vi. Was geht}

(176) Andi : Harry, Was geht? Alles klar?

Der Ausdruck “was geht” ist die Kurzform von "Was geht ab?" und das ist Jugendsprache für "gibt es Neuigkeiten bei dir?". Das Wort hat verschiedene Bedeutungen, nämlich: wie geht's dir (eher nicht so oft, aber kommt vor), als Grü $\beta$ und was machst du gerade?/ gibt was neues. Es ist quasi eine Aufforderung von sich zu erzählen. (www.gutefrage.net).

\section{DIE SCHLUSSFOLGERUNG}

Nach den Untersuchungsergebnissen fallen die Schlussfolgerungen folgendermaßen aus:

1. Es gibt 33 jugendsprachliche Ausdrücke, die im Film "Doktorspiele" von Marco Petry gefunden werden, nämlich: Alter, Assi, Auf jeden, Auf keinsten, Boah, Bock, Blödi, Cool, Echt?, Ey!, Fake, ficken, geil, hammer, hi!, irre, krass, Latte, Mum, Nudelsalat, okay, Party, porno, Schwachmat, spinnen, spitze, spast, super, süß, Typ, voll, was geht?, wow. Es gibt drei unbenutzte Merkmalen der Jugendsprache in diesem Untersuchung, nämlich: Flotte Redensarten und stereotype Floskeln, Metaphorische und Prosodische.

2. Das Ergebnisder Analyse von den Jugendsprachen im Film "Doktorspiele" von Marco Petry wird im fünf Merkmalen der Jugendsprache von Henne kategorisiert, nämlich: eigenwillige Grüße, Anreden und Partnerbezeichnung mit drei Ausdrücken “Alter”, “Ey!'” und “Hi!”, griffige Namen mit zwei Wörter 
https://jurnal.unimed.ac.id/2012/index.php/studia/index

“Blödi” und "Schwachmat”, Entzückungswörter mit nein Ausdrücken "cool”, "geil”, “krass”, “porno”, "spitze”, “irre”, "super”, "süß” und "voll”, Lautwörterkommunikation mit drei Ausdrücken "boah", "echt?" und "wow”, Wortbildungen (Neubedeutungen) mit sechs Wörter "Bock", "hammer", "Latte”, "Nudelsalat”, "Typ” und "spinnen”, Wortbildungen (Neuwörter) mit vier Wörter "cool”, "okay", "fake”und "Party", Wortbildungen (Suffixbildung) mit ein Wort "ficken”, Wortbildungen (Kurzwörter) mit sechs Ausdrücken "Assi”, “Auf jeden”, “Auf keinsten”, "Mum”, “spast” und "was geht”.

\section{LITERATURVERZEICHNIS}

Agustina, A. Chaer. 2010. Sosiolinguitik Perkenalan Awal. Jakarta: Rineka Cipta

Ehmann, Hermann. 2005. Endgeil Das voll korrekte Lexikon der Jugendsprache. München: C.H.Beck oHG.

Eichler, Berit. 2008. Jugendsprache Untersuchung eines komplexen Phänomens im Lichte älterer und neuer Forschungen. Norderstedt Germany: GRIN Verlag

Glass, Birte.2004. Jugendsprache. Norderstedt Germany: GRIN Verlag

Gross, Nicola. 2000. Strukturender heutigen Jugendsprache. Eine soziolinguistische Untersuchung. Norderstedt Germany: GRIN Verlag

Hutagalung, Surya. 2015. Metodologi Penelitian Dalam Praktek. Medan: Unimedpress

Lüttich, Götze. 2004. Grammatik der deutschen Sprache Sprachsystem und Sparachgebrauch. Köln: Karl Müller GmbH

Neuland,Eva. Jugendsprache Eine Einführung.Stuttgart: Narr Francke Attempto Verlag GmbH \& Co.KG

Sumarsono. 2012. Sosiolinguistik. Yogyakarta: Pustaka Belajar

Vogt, Verena. 2017. 100\% Jugendsprache 2017.München: Langenscheidt GmbH \& Co. KG

http://eprints.ums.ac.id/29500/4/BAB_III.pdf, gelesen am 31. March 2017, um 13.15

http://etd.repository.ugm.ac.id-CHAPTER1.pdf, gelesen am 20. March 2017, um 10.45

http://googleweblight.com, gelesen am 12. April 2017, um 10.45

http://image.gala.de, gelesen am 25. Februar 2017, um 19.00 
https://jurnal.unimed.ac.id/2012/index.php/studia/index

http://jurnal.unimed.ac.id, gelesen am 11. Maret 2017, um 19.00

http://lexikon_der_jugendsprache.pdf, gelesen am 5 April 2017, um 11.00

http://lib.unnes.ac.id/2487/1/4645.pdf, gelesen am 25. Februar 2017, um 11.00

www.anglizismen-sprachberatung.de, gelesen am 3 Juli 2017, um 19.00

www.duden.de, gelesn am 3 Juli 2017, um 19.20

www.gutefrage.net, gelesen am 5 Juli 2017, um 21.00

www.mundmische.de, gelesen am 5 Juli 2017, um 21.00

www.redensarten-index.de, gelesen am 3 Juli 2017, um 21.10

www.sprachenudel.de, gelesn am 4 Juli 2017, um 21.20

www.weltbild.de, gelesen am 25. Februar 2017, um 19.00

Biografie des Authors:

Duma Elfrida Manurung ist Alumni des Deutschprogramms von Unimed, Medan 
